\title{
Photoproduction of Hadron Pairs at Fixed-Target Experiments
}

\author{
Christof Hendlmeier ${ }^{1}$, Marco Stratmann ${ }^{2}$ and Andreas Schäfer ${ }^{1}$ \\ 1- Universität Regensburg - Institut für Theoretische Physik, D-93040 Regensburg - Germany \\ 2- Radiation Laboratory - RIKEN, Wako, Saitama 351-0198 - Japan
}

\begin{abstract}
We consider the photoproduction of two hadrons in polarized lepton-nucleon collisions in the framework of perturbative QCD at the next-to-leading order accuracy [1]. After illustrating how to obtain the experimentally relevant observables, a phenomenological study of the photoproduction of hadron pairs at high transverse momenta is presented. We show theoretical predictions for the relevant cross sections at COMPASS and HERMES kinematics as well as theoretical uncertainties.
\end{abstract}

\section{Motivation}

After more than 25 years of studying polarized deep-inelastic lepton-nucleon scattering (DIS) the prime question is still how the proton spin- $\frac{1}{2}$ is composed of the spins and orbital angular momenta of its constituents, quarks and gluons. The single most important result is the finding that quarks spins contribute only little - about $25 \%$ - to the proton spin [2]. The measurement of $\Delta g(x, \mu)$, the polarized gluon distribution of the proton, is the next logical step to clarify the spin puzzle, since it turns out that in the light cone gauge the first moment of $\Delta g(x, \mu)$ has the interpretation of the total contribution of the gluons spin to the proton's spin- $\frac{1}{2}$. The extraction of $\Delta g$ in polarized DIS is, however, very difficult as it contributes only via scaling violations and at higher orders in the strong coupling constant $\alpha_{s}$. Therefore the prime goal of all current experiments with polarized beams is to determine $\Delta g$ directly.

In particular at the Relativistic Heavy Ion Collider (RHIC) at Brookhaven National Laboratory (BNL), many different processes can be studied where $\Delta g$ enters dominantly already at the lowest order (LO) approximation of perturbative QCD (pQCD): for example prompt photon and heavy flavor production, jet and single-inclusive hadron production as well as di-hadron production [3]. First results from the PHENIX and STAR collaboration at RHIC indicate that a large and positive gluon distribution is strongly disfavored in the probed region of momentum fractions $x, 0.03 \lesssim x \lesssim 0.2[4]$.

In addition to RHIC, further information on the spin structure of nucleons can be obtained by fixed-target experiments like COMPASS [5] at CERN or HERMES [6] at DESY. One promising process for the determination of $\Delta g$ at the low energies available at fixedtarget experiments turned out to be the production of hadron pairs at high transverse momenta $p_{T}$ [7]. Some experimental results are already available [8].

\section{Technical framework}

We consider the spin-dependent inclusive photoproduction process

$$
\vec{l}\left(p_{l}\right)+\vec{N}\left(p_{N}\right) \rightarrow l^{\prime}\left(p_{l^{\prime}}\right) H_{c}\left(p_{c}\right) H_{d}\left(p_{d}\right) X,
$$

where a longitudinally polarized lepton $\vec{l}$ with four momentum $p_{l}$ scatters off a longitudinally polarized nucleon $\vec{N}$ with four-momentum $p_{N}$ producing two hadrons $H_{c}$ and $H_{d}$ with 
four momenta $p_{c}$ and $p_{d}$, respectively. The two produced hadrons are assumed to have high transverse momenta $p_{T, c / d}$. Making use of the factorization theorem, the polarized cross section can be written as a convolution of non-perturbative parton distribution and fragmentation functions and hard, short-distance partonic cross sections:

$$
\begin{aligned}
d \Delta \sigma \equiv & \frac{1}{2}\left[d \sigma_{++}-d \sigma_{+-}\right]=\sum_{a b c d} \int d x_{a} d x_{b} d z_{c} d z_{d} \Delta f^{l}\left(x_{a}, \mu_{f}\right) \Delta f^{N}\left(x_{b}, \mu_{f}\right) \times \\
& d \Delta \hat{\sigma}^{a b \rightarrow c d X^{\prime}}\left(S, x_{a}, x_{b}, p_{c} / z_{c}, p_{d} / z_{d}, \mu_{f}, \mu_{f}^{\prime}, \mu_{r}\right) D_{c}^{H_{c}}\left(z_{c}, \mu_{f}^{\prime}\right) D_{d}^{H_{d}}\left(z_{d}, \mu_{f}^{\prime}\right) .
\end{aligned}
$$

The sum runs over all possible partonic channels $a b \rightarrow c d$ with $d \Delta \hat{\sigma}^{a b \rightarrow c d X^{\prime}}$ the relevant, perturbatively calculable hard partonic cross sections at next-to-leading order (NLO) accuracy. The subscripts $(++)$ and $(+-)$ denote the helicities of the lepton beam and the nucleon target at rest. $S$ is the total center-of-mass system (c.m.s.) energy, i.e. $S=\left(p_{l}+p_{N}\right)^{2}$. The $\Delta f^{N}\left(x_{b}, \mu_{f}\right)$ are the usual spin-dependent parton densities for parton $b$ in a nucleon at a momentum fraction $x_{b}$ and scale $\mu_{f} \cdot D_{c / d}^{H_{c / d}}\left(z_{c / d}, \mu_{f}^{\prime}\right)$ describe the fragmentation of a parton $c / d$ into a hadron $H_{c / d}$ at a momentum fraction $z_{c / d}$ and scale $\mu_{f}^{\prime} . \Delta f^{l}\left(x_{a}, \mu_{f}\right)$ represents the spin-dependent Weizsäcker-Williams equivalent photon spectrum [9] describing the collinear radiation of a photon with momentum fraction $x_{a}$ and virtuality lower than some upper value $Q_{\max }^{2}$. All phenomenological studies have been done for the so-called direct case, where the photon interacts directly with a parton of the nucleon. No resolved photon contributions are included so far. For a proper treatment of the collinear, infrared and ultraviolet divergencies appearing in NLO calculations of the hard partonic cross sections we introduced a variable $z$ defined by

$$
z \equiv-\frac{\vec{p}_{T, c} \cdot \vec{p}_{T, d}}{p_{T, c}^{2}}
$$

in a system in which the incoming beam defines the longitudinal axis. For a covariant definition of the variable $z$ and some technical details we refer to a work by Aurenche et al. [10]. To guarantee that the two hadrons are in opposite hemispheres, we restrict ourselves to the range $z>0$. Needless to say, the required spin-averaged cross section $d \sigma$ is straightforwardly obtained by replacing all polarized quantities in Eq. (2) by their appropriate unpolarized counterparts.

\section{Phenomenological results}

In our phenomenological studies we concentrate on the production of charged hadrons made of light quark flavors. We sum over pions, kaons, and (anti-)protons and use fragmentation functions of KKP [11]. For parton distributions we employ the unpolarized CTEQ6M [12] and polarized GRSV standard sets [13] as well as the sets of DNS [14]. If it is not stated otherwise, the factorization/renormalization scales in Eq. (2) are all set equal $\mu \equiv \mu_{f}=$ $\mu_{f}^{\prime}=\mu_{r}=p_{T, c}+p_{T, d}$. All NLO results presented in this paper are preliminary.

\subsection{Two-hadron production at COMPASS}

At the COMPASS experiment at CERN polarized muons are scattered with a beam energy of $E_{\mu}=160 \mathrm{GeV}$ off the deuterons in a polarized ${ }^{6} \mathrm{LiD}$ solid-state target corresponding to a c.m.s. energy of $\sqrt{S} \simeq 18 \mathrm{GeV}$. For the calculations we demand that hadron $H_{c}$ has a 


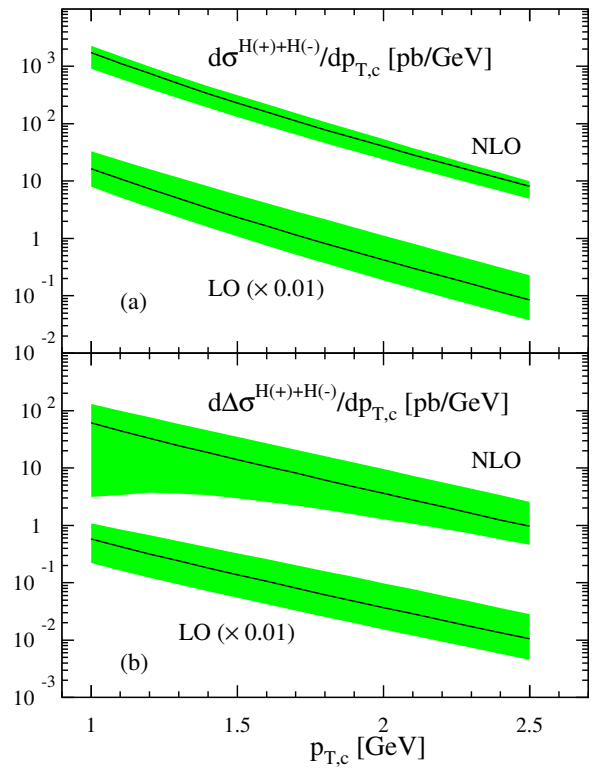

Figure 1: Scale dependence at COMPASS kinematics. is reduced in the unpolarized cross section. This might indicate that we are in a regime where perturbative QCD is applicable. However no improvement is observed in the polarized case and in both the unpolarized and polarized case for lower z-cuts $(z>0.2,0.4)$. As a consequence the applicability of the perturbative approach can not be taken for granted. An important benchmark for testing the pQCD framework would be, for instance, to check if the unpolarized data fall within the uncertainty band, where the parton distributions are already known very well.

Once the applicability of pQCD is established, the double spin asymmetry turns out to be very sensitive to the gluon polarization assumed in the calculation. Varying from maximal positive to maximal negative sets of GRSV the asymmetry is in the range $-0.1 \lesssim A_{L L} \lesssim 0.4$ which would allow at least a determination of the sign of $\Delta g$.

\subsection{Two-hadron production at HERMES}

At the HERMES experiment at DESY a longitudinally polarized electron (positron) beam with $E_{e} \simeq 27.5 \mathrm{GeV}$ is scattered off a proton or deuterium gas target. We concentrate on results for a deuterium target as this has the better statistics. The corresponding c.m.s. energy is $\sqrt{S} \simeq 7.25 \mathrm{GeV}$. We choose a maximal photon virtuality of $Q_{\max }^{2}=0.1 \mathrm{GeV}^{2}$ and restrict the momentum fraction $x_{a}$ of the lepton carried by the produced photon to $0.2 \leq x_{a} \leq 0.9$. For hadron $H_{c}$ we use an acceptance cut of $40 \mathrm{mrad}<\theta_{\text {lab }}<220 \mathrm{mrad}$. The fraction of the parton's momenta carried by the produced hadrons are restricted to the range $z_{c}, z_{d} \geq 0.1$. 
Figure 2 shows the scale dependence of the unpolarized (a) and polarized (b) cross sections as a function of the transverse momentum of one hadron $p_{T, c}$ when varying the scales in the range $1 / 2\left(p_{T, c}+p_{T, d}\right) \leq \mu \leq$ $2\left(p_{T, c}+p_{T, d}\right)$. Due to the smaller c.m.s. energy there is no noticeable reduction of the scale dependence when going to NLO accuracy. We observed the same for other $z$-cuts both for unpolarized and polarized case. We emphasize that all comments about applicability of pQCD also apply here. Again, as it is for COMPASS kinematics, the double spin asymmetry $A_{L L}$ is very sensitive to the chosen $\Delta g$ polarization: $-0.1 \lesssim A_{L L} \lesssim 0.5$ when varying between $\Delta g=g$ and $\Delta g=-g$ at the input scale with $g$ the unpolarized gluon distribution in the nucleon.

\section{Acknowledgments}

C.H. was supported by a grant of the "Bayerische Eliteförderung". This work was supported in part by the "Deutsche Forschungs-

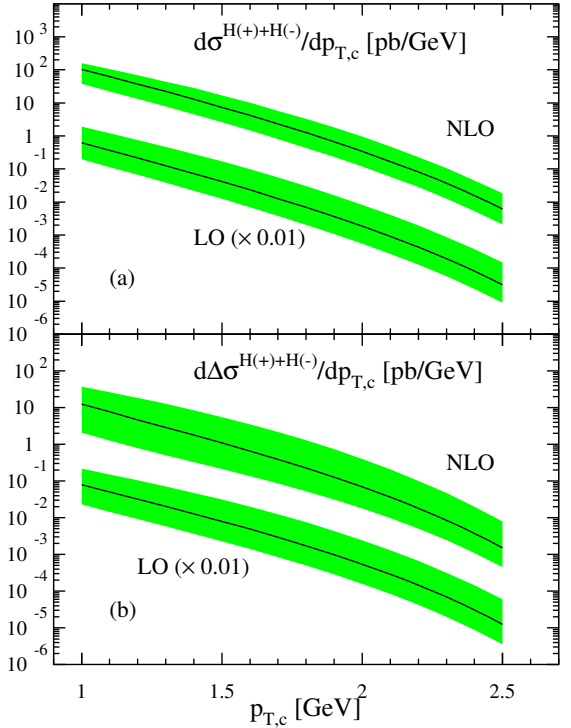

Figure 2: Scale dependence at HERMES kinematics. gesellschaft (DFG)".

\section{References}

[1] Slides: http://indico . cern. ch/contributionDisplay $\cdot$ py? contribId=138\&sessionId=4\&conf Id=9499

[2] See, for example: K. Rith, in Proceedings of the 12th International Workshop on Deep Inelastic Scattering (DIS 2004), Strbske Pleso, Slovakia, D. Bruncko et al. (eds.), p. 138, (2004).

[3] See, for example: G. Bunce, N. Saito, J. Soffer and W. Vogelsang, Ann. Rev. Nucl. Part. Sci. 50525 (2000); C. Aidala et al., Research Plan for Spin Physics at RHIC, BNL report BNL-73798-2005, (2005).

[4] PHENIX Collaboration, S.S. Adler et al., Phys. Rev. Lett. 93202002 (2004); Phys. Rev. D73 091102 (2006); K. Boyle, AIP Conf. Proc. 842351 (2006); STAR Collaboration, B.I. Abelev et al., Phys. Rev. Lett. 97252001 (2006).

[5] COMPASS Collaboration, G. Baum et al., CERN/SPSLC 96-14 (1996).

[6] HERMES Collaboration, The HERMES Physics Program \& Plans for 2001-2006, DESY-PRC, 2000.

[7] A. Bravar, D. von Harrach and A. Kotzinian, Phys. Lett. B421 349 (1998).

[8] HERMES Collaboration, A. Airapetian et al., Phys. Rev. Lett. 842584 (2000); Spin Muon Collaboration (SMC), B. Adeva et al., Phys. Rev. D70 012002 (2004); COMPASS Collaboration, E.S. Ageev et al. Phys. Lett. B633 25 (2006).

[9] D. de Florian and S. Frixione, Phys. Lett. B457 236 (1999).

[10] P. Aurenche et al., Z. Phys. C24 309 (1984); Z. Phys. C29 459 (1985).

[11] B.A. Kniehl et al., Nucl. Phys. B582 541 (2000).

[12] CTEQ Collaboration, J. Pumplin et al., JHEP 0207012 (2002).

[13] M. Glück, E. Reya, M. Stratmann and W. Vogelsang, Phys. Rev. D63 094005 (2001).

[14] D. de Florian, G.A. Navarro and R. Sassot, Phys. Rev. D71 094018 (2005).

[15] C. Hendlmeier, M. Stratmann and A. Schäfer, Eur. Phys. J. C48 135 (2006). 